\title{
Linear relations among four-point functions in the high energy limit of string theory
}

\author{
Pei-Ming Ho* and Xue-Yan Lin $^{\dagger}$ \\ Department of Physics, National Taiwan University, Taipei, Taiwan, Republic of China
}

(Received 3 May 2006; published 22 June 2006)

\begin{abstract}
The decoupling of spurious states leads to linear relations among four-point functions in the highenergy limit of string theory. Recently it was shown that the linear relations uniquely determine ratios among four-point functions at the leading order. The purpose of this paper is to extend the validity of the same approach to the next-to-leading order and higher orders.
\end{abstract}

DOI: 10.1103/PhysRevD.73.126007

PACS numbers: 11.25.- w, 11.25.Mj, 11.25.Sq

\section{MOTIVATION}

Many have posed the question of whether there is a fundamental principle underlying string theory, in a way analogous to how the equivalence principle led Einstein to general relativity. For example, for the bosonic open string theory, Witten's cubic string field theory is formally a Chern-Simons theory with infinite gauge symmetry, but the gauge symmetry of the infinitely many massive higher spin gauge fields is not manifest in the flat background. It is tempting to imagine that a Higgs mechanism is responsible for the masses of the gauge fields, and the symmetry will be restored when we consider physics at sufficiently high energies. Gross and Mende [1], Gross [2], and Gross and Manes [3] explored this possibility for bosonic open and closed strings. They used the saddle point approximation to evaluate the integral over moduli space for four-point functions. They found that, to the 0th order approximation in the high-energy limit, the saddle point is independent of the particles participating in the scattering, and claimed that this is a signal of the hidden symmetry. Unfortunately, it was shown later [4-6] that some of their results are incorrect. On the other hand, it is not clear how the existence of a universal 0th order saddle point is related to the existence of symmetry.

Following a series of earlier works [4-13], our approach is to explore the implication of the decoupling of spurious states from other physical states. Spurious states correspond to gauge symmetries. One of the most salient features of string theory is that it has infinitely many higher spin gauge fields with infinitely many gauge symmetries. There is a delicate conspiracy among all the fields and symmetries so that, in spite of many no-go theorems and folklore about interacting higher spin gauge fields, string theory is a consistent interacting theory of massive higher spin gauge fields in flat spacetime. In view of this mystery it is worthwhile to study spurious states in detail.

In [4-6,9-13], the decoupling of spurious states is used to derive linear relations among four-point functions at the leading order in the high-energy limit. In the old covariant

\footnotetext{
*Electronic address: pmho@phys.ntu.edu.tw

†Electronic address: xueyan.lin@msa.hinet.net
}

first quantized formulation of string theory, spurious states represent gauge transformations. Normally, on-shell gauge transformations only transform a state to itself in a different gauge, and never transform a state to another different state. The crucial step which made it possible to relate physically inequivalent states is that, in the high-energy limit, we ignore subleading terms in the gauge transformation, and the spurious states are no longer exactly orthogonal to all physical states. It turns out that the many gauge transformations intertwine and overlap with each other so much that the assumption of a smooth, consistent highenergy limit of string theory uniquely fixes ratios among four-point functions to the leading order. Remarkably, via simple algebraic manipulations, numerical ratios among four-point functions were obtained explicitly for all mass levels [9-11]. These ratios involve all four-point functions at the leading order in the high-energy limit.

The purpose of this paper is to extend our understanding to the next-to-leading order. The first question is whether amplitudes at the next-to-leading order are also unique up to an overall constant at all mass levels. Our answer is yes. We also claim that there are linear relations at higher orders (see Sec. VI), although they are not sufficient to fix all amplitudes of that order to be proportional to each other.

There are other approaches in the literature which are also based on studies of the algebraic structure of the string worldsheet theory, such as $[14,15]$. Our approach distinguishes itself by giving the simplest and most explicit relations among correlation functions. People have also tried to define tensionless strings [16] to describe strings in the high-energy limit, as well as to construct various higher spin gauge theories [17] to mimic string theory. These approaches illuminate different aspects of the problem and suggest answers in different directions. Hopefully we will be able to make connections with these other approaches to have a better understanding of the hidden symmetry.

\section{PRELIMINARIES AND CONVENTIONS}

The focus of this paper is on four-point functions at the next-to-leading order. Three-point functions are trivial in the sense that we can not take a high-energy limit without going off shell, and there is no parameter other than the 
center of mass energy so that all amplitudes of the same order are trivially identical up to a constant factor. On the other hand, five-point functions depend on too many parameters and so the linear relations are insufficient to determine their ratios uniquely.

For the scattering process of two incoming and two outgoing particles, the four momenta define a $2+1$ dimensional scattering plane. Because of the Poincaré symmetry, the scattering amplitude only depends on two parameters. We choose them to be $E$ and $\phi . E$ is the center of mass energy $\left(s=4 E^{2}\right)$, and $\phi$ is the scattering angle between the momenta of the 1st and 3rd particles in the center of mass frame.

The scattering amplitude is given by a four-point correlation function. The high-energy limit we consider is

$$
E \rightarrow \infty, \quad \phi=\text { fixed. }
$$

That is, we compute four-point functions and keep only the first nonvanishing terms in the $1 / E$ expansion.

For a particle of mass $M$ and momentum

$$
P=(E, k, 0, \cdots, 0),
$$

we will use the following basis of polarizations:

$$
\begin{gathered}
e^{P}=(E / M, k / M, 0, \cdots, 0), \\
e^{L}=(k / M, E / M, 0, \cdots, 0), \\
e^{T}=(0,0,1, \cdots, 0),
\end{gathered}
$$

and call them the momentum, longitudinal, and transverse polarizations, respectively. These constitute the basis of vectors on the scattering plane. We will use $e^{I}$ to denote unit vectors perpendicular to the scattering plane.

When we compare four-point functions, we fix three of the four vertices (say $V_{2}, V_{3}, V_{4}$ ), and let only one vertex $\left(V_{1}\right)$ be different. Each polarization vector $\left(e^{T}, e^{L}, e^{I}\right)$ of the vertex operator

$$
V_{1}\left(k_{1}\right)=\left[\left(\partial X^{L}\right)^{m}\left(\partial X^{T}\right)^{n}\left(\partial X^{I}\right)^{q} \cdots\right] e^{i k_{1} \cdot X},
$$

which corresponds to the state

$$
\left(\alpha_{-1}^{L}\right)^{m}\left(\alpha_{-1}^{T}\right)^{n}\left(\alpha_{-1}^{I}\right)^{q} \cdots|0 ; k\rangle,
$$

will have to be contracted with another vector to form a Lorentz invariant in the expression of the scattering amplitude. In the high-energy limit, the amplitude is dominated by contractions with momenta $k_{i}$. The polarizations $e^{I}$ perpendicular to the scattering plane are kinematically suppressed as their contractions with $k_{i}$ 's vanish. The timelike polarization $e^{P}$ can also be avoided as a choice of gauge fixing. Therefore, we will focus our attention on only two polarizations, $e^{L}$ and $e^{T}$.

At the leading order in the $1 / E$ expansion of the fourpoint function, in addition to the common factor resembling the four-tachyon amplitude, each factor of $\partial^{n} X^{\mu}$ in the vertex operator contributes a certain power of $E$ to the scattering amplitude. We have [9]

$$
\begin{gathered}
\left(\alpha_{-1}^{L}\right)^{2 m} \sim E^{2 m}, \\
\left(\alpha_{-1}^{L}\right)^{2 m+1} \sim E^{2 m}, \\
\alpha_{-n}^{L} \sim E^{2}, \quad n \geq 2, \\
\alpha_{-n}^{T} \sim E^{1}, \quad n \geq 1 .
\end{gathered}
$$

In general, when we compare four-point functions for different $V_{1}$ 's at the same mass level, the highest spin state $\left(\alpha_{-1}^{T}\right)^{n}|0 ; k\rangle$ is always at the leading order. There are more and more other states at the leading order when we go to higher and higher mass levels. They are all of the form [9]

$$
\left(\alpha_{-1}^{L}\right)^{2 m}\left(\alpha_{-1}^{T}\right)^{n}\left(\alpha_{-2}^{L}\right)^{q}|0 ; k\rangle .
$$

Ratios between two four-point functions at the leading order are uniquely fixed by the requirement that spurious states be decoupled from all physical states, assuming that string theory has a smooth high-energy limit. The master equation which gives the ratio between any two leadingorder amplitudes (for $V_{1}$ 's at the same mass level) is [9-11]

$$
\begin{gathered}
\lim _{E \rightarrow \infty} \frac{\mathcal{T}\left(\left|V_{1}\right\rangle=\left(\alpha_{-1}^{T}\right)^{n-2 m-2 q}\left(\alpha_{-1}^{L}\right)^{2 m}\left(\alpha_{-2}^{L}\right)^{q}\left|0, k_{1}\right\rangle\right)}{\mathcal{T}\left(\left|V_{1}\right\rangle=\left(\alpha_{-1}^{T}\right)^{n}\left|0 ; k_{1}\right\rangle\right)} \\
=\left(\frac{-1}{m_{1}}\right)^{2 m+q}\left(\frac{1}{2}\right)^{m+q}(2 m-1) ! !
\end{gathered}
$$

The same result can also be derived from Virasoro constraints.

The linear relations found before and those that will be derived here apply to different choices of $V_{1}$ at the same mass level, and are independent of the choices of $V_{2}, V_{3}$, $V_{4}$. When we say that an amplitude is at the leading order, we mean that it is among the most dominant amplitudes in the high-energy limit for all possible choices of $V_{1}$ at the same mass level (without changing $V_{2}, V_{3}, V_{4}$ ). Equation (13) says that the $\phi$ dependence of the leadingorder terms in the $1 / E$ expansion of all leading-order amplitudes is the same (for a given mass level), and the numerical ratios can be uniquely determined by requiring the decoupling of spurious states.

In this paper we focus on the bosonic open string theory. Our result can be immediately applied to bosonic closed strings, whose amplitudes factorize into open string amplitudes. It should also be possible to extend our results to superstrings. Definitions of the kinematic variables of fourpoint functions are given in the Appendix, and we will use the convention that $\alpha^{\prime}=1 / 2$.

\section{FIRST MASSIVE LEVEL $\left(M^{2}=2\right)$}

In this section we take the first massive level as an example to review earlier results [4-6]. Readers familiar with these results should skip to the next section. 
Physical states in the first massive level are

$$
\left(\epsilon_{\mu \nu} \alpha_{-1}^{\mu} \alpha_{-1}^{\nu}+\epsilon_{\mu} \alpha_{-2}^{\mu}\right)|0 ; k\rangle,
$$

where the parameters $\epsilon_{\mu \nu}, \epsilon_{\mu}$, and $k_{\mu}$ satisfy

$$
\begin{gathered}
k^{2}=-M^{2}=-2, \quad 2 \epsilon_{\mu \nu} k^{\nu}+2 \epsilon_{\mu}=0, \\
\epsilon_{\mu}^{\mu}+2 \epsilon_{\mu} k^{\mu}=0 .
\end{gathered}
$$

There are two sets of spurious states,

$$
\begin{aligned}
& L_{-1} \alpha_{-1}^{\mu}|0 ; k\rangle=\left(p_{\nu} \alpha_{-1}^{\mu} \alpha_{-1}^{\nu}+\alpha_{-2}^{\mu}\right)|0 ; k\rangle, \\
& L_{-2}|0 ; k\rangle=\left(\frac{1}{2} \eta_{\mu \nu} a_{-1}^{\mu} \alpha_{-1}^{\nu}+p_{\mu} \alpha_{-2}^{\mu}\right)|0 ; k\rangle .
\end{aligned}
$$

Some states are both spurious and physical; they have zero norm and are called zero-norm states. There are two sets of zero-norm states at this mass level,

$$
\begin{gathered}
\left(\Lambda_{(\mu} p_{\nu)} a_{-1}^{\mu} \alpha_{-1}^{\nu}+\Lambda_{\mu} \alpha_{-2}^{\mu}\right)|0 ; k\rangle, \quad \Lambda \cdot p=0, \\
\lambda\left[\left(\frac{1}{2} \eta_{\mu \nu}+\frac{3}{2} p_{\mu} p_{\nu}\right) a_{-1}^{\mu} \alpha_{-1}^{\nu}+\frac{5}{2} p_{\mu} \alpha_{-2}^{\mu}\right]|0 ; k\rangle .
\end{gathered}
$$

The first step of our approach is to demand zero-norm states to be decoupled from physical states,

$$
\mathcal{T} \equiv\left\langle V_{1}\left(k_{1}\right) V_{2}\left(k_{2}\right) V_{3}\left(k_{3}\right) V_{4}\left(k_{4}\right)\right\rangle=0,
$$

where $V_{1}$ is the vertex operator of a zero-norm state, while $V_{2}, V_{3}$, and $V_{4}$ are vertex operators of three arbitrary physical states. Taking $V_{1}$ to be at this mass level, and thus of the form (14), we can decompose $\mathcal{T}$ as

$$
\mathcal{T}=\epsilon_{\mu \nu} \mathcal{T}^{\mu \nu}+\epsilon_{\mu} \mathcal{T}^{\mu},
$$

where $\mathcal{T}^{\mu \nu}$ and $\mathcal{T}^{\mu}$ are the correlation functions with $V_{1}$ being the basis states $\alpha_{-1}^{\mu} \alpha_{-1}^{\nu}|0 ; k\rangle$ and $\alpha_{-2}^{\mu}|0 ; k\rangle$,

$$
\begin{aligned}
\mathcal{T}^{\mu \nu} & \leftrightarrow V_{1}=\partial X^{\mu} \partial X^{\nu} e^{i k_{1} \cdot X}, \\
\mathcal{T}^{\mu} & \leftrightarrow V_{1}=\partial^{2} X^{\mu} e^{i k_{1} \cdot X} .
\end{aligned}
$$

Strictly speaking, $\mathcal{T}^{\mu \nu}$ and $\mathcal{T}^{\mu}$ are not well defined because only physical states admit a path integral independent of the gauge-fixing condition. Hence we should restrict our attention to those linear combinations that correspond to physical states.

The decoupling of the zero-norm states (18) and (19) from other physical states implies that

$$
\begin{gathered}
\sqrt{2} \mathcal{T}^{L P}+\mathcal{T}^{L}=0, \\
\sqrt{2} \mathcal{T}^{T P}+\mathcal{T}^{T}=0, \\
\mathcal{T}^{\mu}{ }_{\mu}+6 \mathcal{T}^{P P}+5 \sqrt{2} \mathcal{T}^{P}=0,
\end{gathered}
$$

where $P, L, T$ stand for contraction with the polarization vectors $e^{P}, e^{L}, e^{T}$, respectively. We will refer to these relations as Ward identities.

For higher and higher mass levels, zero-norm states are more and more complicated, and so are the corresponding
Ward identities. But since we will only focus on the highenergy limit, there is an easier way to derive the Ward identities. When we take the high-energy limit of a zeronorm state, it will not be of zero norm anymore because we will ignore higher order components in the $1 / E$ expansion. Thus we should simply consider the decoupling of the spurious states. The Ward identities derived from spurious states are

$$
\begin{gathered}
\sqrt{2} \mathcal{T}^{\mu P}+\mathcal{T}^{\mu}=0, \\
\frac{1}{2} \mathcal{T}^{\mu}{ }_{\mu}+\sqrt{2} \mathcal{T}^{P}=0 .
\end{gathered}
$$

Notice that Eqs. (23)-(25) are linear combinations of Eqs. (26) and (27). The spurious states constitute a larger symmetry because they lead to a Ward identity $\left(\sqrt{2} \mathcal{T}^{P P}+\right.$ $\mathcal{T}^{P}=0$ ) which does not exist in Eqs. (23)-(25).

Now we assume that scattering amplitudes remain the same at the leading order in the high-energy limit under the replacement

$$
e^{P} \rightarrow e^{L} .
$$

Naively, this seems a direct result of the fact that $e^{P}-$ $e^{L}=\mathcal{O}(1 / E)$. However, if the scattering amplitude does not have a smooth high-energy limit, this assumption may not be correct. The validity of the replacement (28) is an assertion of the smoothness of the high-energy limit of string theory [6].

Under the replacement (28), Eqs. (26) and (27) become

$$
\begin{gathered}
\sqrt{2} \mathcal{T}^{L P}+\mathcal{T}^{L}=0 \quad \rightarrow \quad \sqrt{2} \mathcal{T}^{L L}+\mathcal{T}^{L}=0, \\
\sqrt{2} \mathcal{T}^{T P}+\mathcal{T}^{T}=0 \quad \rightarrow \quad \sqrt{2} \mathcal{T}^{T L}+\mathcal{T}^{T}=0, \\
\frac{1}{2}\left(-\mathcal{T}^{P P}+\mathcal{T}^{L L}+\mathcal{T}^{T T}\right)+\sqrt{2} \mathcal{T}^{P}=0 \\
\rightarrow \quad \frac{1}{2} \mathcal{T}^{T T}+\sqrt{2} \mathcal{T}^{L}=0 .
\end{gathered}
$$

Here we used the fact that transverse polarizations $e^{I}$ are kinematically suppressed to rewrite $\mathcal{T}^{\mu}{ }_{\mu}$ as $-\mathcal{T}^{P P}+$ $\mathcal{T}^{L L}+\mathcal{T}^{T T}$. (After replacing $P$ by $L, \mathcal{T}^{\mu}{ }_{\mu}$ becomes just $\mathcal{T}^{T T}$.)

For $M^{2}=2$, the physical amplitudes at the leading order are $\mathcal{T}^{L L}$ and $\mathcal{T}^{T T}$. Note that, although $\alpha_{-1}^{L} \alpha_{-1}^{L}|0 ; k\rangle$ and $\alpha_{-1}^{T} \alpha_{-1}^{T}|0 ; k\rangle$ are not physical states, they can be extended into physical states without changing the four-point function,

$$
\begin{aligned}
& \left(\alpha_{-1}^{L} \alpha_{-1}^{L}-\alpha_{-1}^{I} \alpha_{-1}^{I}\right)|0 ; k\rangle \rightarrow \mathcal{T}^{L L}, \\
& \left(\alpha_{-1}^{T} \alpha_{-1}^{T}-\alpha_{-1}^{I} \alpha_{-1}^{I}\right)|0 ; k\rangle \rightarrow \mathcal{T}^{T T} .
\end{aligned}
$$

From Eqs. (29) and (31), we expect $\mathcal{T}^{L L}$ and $\mathcal{T}^{T T}$ to have the ratio 1:4 in the high-energy limit. By directly calculating the exact amplitudes and expanding them in powers of $1 / E$, we can verify this result. As an example, the exact four-point function $\mathcal{T}^{\mu \nu}$ with $V_{2}, V_{3}, V_{4}$ corre- 
sponding to three tachyons is

$$
\mathcal{T}^{\mu \nu}=\int_{-\infty}^{\infty} \prod_{i=1}^{4} d x_{i}\left\langle: \partial X^{\mu} \partial X^{\nu} e^{i k_{1} \cdot X}:: e^{i k_{2} \cdot X}:: e^{i k_{3} \cdot X}:: e^{i k_{4} \cdot X}:\right\rangle
$$

$$
\begin{aligned}
= & \left(\frac{\Gamma\left(\frac{-s}{2}-1\right) \Gamma\left(\frac{-t}{2}-1\right)}{\Gamma\left(\frac{u}{2}+2\right)}+\frac{\Gamma\left(\frac{-t}{2}-1\right) \Gamma\left(\frac{-u}{2}-1\right)}{\Gamma\left(\frac{s}{2}+2\right)}\right. \\
& \left.+\frac{\Gamma\left(\frac{-u}{2}-1\right) \Gamma\left(\frac{-s}{2}-1\right)}{\Gamma\left(\frac{t}{2}+2\right)}\right)\left[s / 2(s / 2+1) k_{3}^{\mu} k_{3}^{\nu}\right. \\
& \left.-2(s / 2+1)(u / 2+1) k_{2}^{(\mu} k_{3}^{\nu)}+u / 2(u / 2+1) k_{2}^{\mu} k_{2}^{\nu}\right] \\
& +\left(k_{3} \leftrightarrow k_{4}\right),
\end{aligned}
$$

where $s=-\left(k_{2}+k_{1}\right)^{2}, \quad t=-\left(k_{2}+k_{3}\right)^{2}, \quad$ and $\quad u=$ $-\left(k_{2}+k_{4}\right)^{2}$.

We can now calculate all amplitudes in Eq. (35). After some algebra, we get

$$
\begin{gathered}
\mathcal{T}^{L L}=\mathcal{T}(2) E^{6}\left[2 \sin ^{2} \phi+O\left(\frac{1}{E^{4}}\right)\right], \\
\mathcal{T}^{T T}=\mathcal{T}(2) E^{6}\left[8 \sin ^{2} \phi+20 \sin ^{2} \phi \frac{1}{E^{2}}+O\left(\frac{1}{E^{4}}\right)\right], \\
\mathcal{T}^{L T}=\mathcal{T}(2) E^{5}\left[4 \sqrt{2} \cos \phi \sin \phi+6 \sqrt{2} \cos \phi \sin \phi \frac{1}{E^{2}}\right. \\
\left.+O\left(\frac{1}{E^{4}}\right)\right],
\end{gathered}
$$

where

$$
\begin{aligned}
\mathcal{T}(2)= & \frac{\Gamma\left(\frac{-s}{2}-1\right) \Gamma\left(\frac{-t}{2}-1\right)}{\Gamma\left(\frac{u}{2}+2\right)}+\frac{\Gamma\left(\frac{-t}{2}-1\right) \Gamma\left(\frac{-u}{2}-1\right)}{\Gamma\left(\frac{s}{2}+2\right)} \\
& +\frac{\Gamma\left(\frac{-u}{2}-1\right) \Gamma\left(\frac{-s}{2}-1\right)}{\Gamma\left(\frac{t}{2}+2\right)} .
\end{aligned}
$$

We find that indeed $\mathcal{T}^{L L}: \mathcal{T}^{T T}=1: 4$ in the high-energy limit.

At this mass level, $\mathcal{T}^{L L}$ and $\mathcal{T}^{T T}$ are the only physical amplitudes at the leading order. All other physical states are either related to them via zero-norm states, or are at a lower order.

It is interesting that Eqs. (29) and (31) also determine $\mathcal{T}^{L}$ to have a fixed ratio with $\mathcal{T}^{L L}$ and $\mathcal{T}^{T T}$,

$$
\mathcal{T}^{L L}: \mathcal{T}^{T T}: \mathcal{T}^{L}=1: 4:-\sqrt{2},
$$

although $\mathcal{T}^{L}$ does not correspond to a physical state. Under conformal transformations, the path integral is not invariant for nonphysical states, so it seems weird to predict a fixed relation involving $\mathcal{T}^{L}$. The reason is that the conformal anomaly of $\mathcal{T}^{L}$ appears only at the subleading $\operatorname{order}\left(\frac{1}{E^{2}}\right)$. On the other hand, for the amplitude $\mathcal{T}^{T}$, which is at a lower order than $\mathcal{T}^{L}$, anomaly occurs at its leading order, so it cannot have a well-defined value.

At this mass level, there is only one amplitude $\mathcal{T}^{L T}$ at the next-to-leading order, so we need to look at higher mass levels.

\section{SECOND MASSIVE LEVEL $\left(M^{2}=4\right)$}

The details of computation for the second massive level $\left(M^{2}=4\right)$ are similar to that for the first massive level in the previous section. We simply list the results here.

The physical states of interest are

$$
\begin{gathered}
|A\rangle=\left[\left(\alpha_{-1}^{T}\right)^{3}-3\left(\alpha_{-1}^{I}\right)^{2} \alpha_{-1}^{T}\right]|0 ; k\rangle, \\
|B\rangle=\left[\alpha_{-1}^{T}\left(\alpha_{-1}^{L}\right)^{2}-\alpha_{-1}^{T}\left(\alpha_{-1}^{I}\right)^{2}\right]|0 ; k\rangle, \\
|C\rangle=\left[\alpha_{-1}^{L}\left(\alpha_{-1}^{T}\right)^{2}-\alpha_{-1}^{L}\left(\alpha_{-1}^{I}\right)^{2}\right]|0 ; k\rangle, \\
|D\rangle=\left[\left(\alpha_{-1}^{L}\right)^{3}-3 \alpha_{-1}^{L}\left(\alpha_{-1}^{I}\right)^{2}\right]|0 ; k\rangle, \\
|E\rangle=\frac{1}{2}\left[\alpha_{-1}^{T} \alpha_{-2}^{L}-\alpha_{-1}^{L} \alpha_{-2}^{T}\right]|0 ; k\rangle .
\end{gathered}
$$

The 2nd terms on the right-hand side can be ignored in the high-energy limit.

With the following notation for amplitudes associated with different choices of $V_{1}$,

$$
\begin{gathered}
\mathcal{T}^{\mu \nu \lambda} \leftrightarrow \alpha_{-1}^{\mu} \alpha_{-1}^{\nu} \alpha_{-1}^{\lambda}|0 ; k\rangle, \\
\mathcal{T}^{\mu \nu} \leftrightarrow \alpha_{-1}^{\mu} \alpha_{-2}^{\nu}|0 ; k\rangle,
\end{gathered}
$$

$$
\mathcal{T}^{\mu} \leftrightarrow \alpha_{-3}^{\mu}|0 ; k\rangle,
$$

the decoupling of spurious states implies, after replacing $P$ by $L$,

$$
\begin{gathered}
\mathcal{T}^{L T T}+\mathcal{T}^{T T}=0, \\
\mathcal{T}^{L L T}+\mathcal{T}^{(L T)}=0, \\
\mathcal{T}^{L L L}+\mathcal{T}^{L L}=0, \\
\mathcal{T}^{L T}+\mathcal{T}^{T}=0, \\
\mathcal{T}^{L L}+\mathcal{T}^{L}=0, \\
\mathcal{T}^{T T}+2 \mathcal{T}^{L}=0, \\
\frac{1}{2} \mathcal{T}^{T T T}+2 \mathcal{T}^{T L}+\mathcal{T}^{T}=0, \\
\frac{1}{2} \mathcal{T}^{L T T}+2 \mathcal{T}^{L L}+\mathcal{T}^{L}=0,
\end{gathered}
$$

where $\mathcal{T}^{(L T)} \equiv \frac{1}{2}\left(\mathcal{T}^{L T}+\mathcal{T}^{T L}\right)$. 
Together with some rough power counting, these linear relations allow us to determine which amplitudes are at the leading order, as well as their ratios. It turns out that the states $|A\rangle,|B\rangle$, and $|E\rangle$ are at the leading order and have the ratios [4]

$$
\mathcal{T}(A): \mathcal{T}(B): \mathcal{T}(E)=8: 1:-1
$$

The ratios can be derived algebraically from Eqs. (49)(56). Similar to the case of $M^{2}=2$, there is another nonphysical state $\left(\left[\alpha_{-1}^{T} \alpha_{-2}^{L}+\alpha_{-1}^{L} \alpha_{-2}^{T}\right]|0 ; k\rangle\right)$ at $M^{2}=4$ which is linearly related to these states at the leading order.

What is new is that the amplitudes at the next-to-leading order are also identical up to numerical constant factors

$$
\mathcal{T}(C): \mathcal{T}(D)=2: 1
$$

This ratio can be solved from the Ward identities (51), (53), and (56) above. Unlike the leading-order case, only physical amplitudes have fixed ratios with each other. Because of this difference, it is much harder to derive a master formula for the ratio between any two next-to-leadingorder amplitudes at arbitrary mass levels, since we do not have general formulas for physical states at arbitrary mass levels.

Utilizing the ratios among the leading-order amplitudes, one can construct physical states whose four-point functions are next to the next-to-leading order,

$$
(|A\rangle-8|B\rangle), \quad(|E\rangle+2|B\rangle) .
$$

Unfortunately, the ratio of their amplitudes is not a constant.

\section{THIRD MASSIVE LEVEL $\left(M^{2}=6\right)$}

Analogous to the previous two cases, we list all the physical states that we should consider:

$$
\begin{gathered}
|A\rangle=\left[\left(\alpha_{-1}^{T}\right)^{4}-6\left(\alpha_{-1}^{T}\right)^{2}\left(\alpha_{-1}^{I}\right)^{2}+\left(\alpha_{-1}^{I}\right)^{4}\right]|0 ; k\rangle \\
|B\rangle=\left[\left(\alpha_{-1}^{T}\right)^{3} \alpha_{-1}^{L}-3 \alpha_{-1}^{T} \alpha_{-1}^{L}\left(\alpha_{-1}^{I}\right)^{2}\right]|0 ; k\rangle \\
|C\rangle=\left[\left(\alpha_{-1}^{T}\right)^{2}\left(\alpha_{-1}^{L}\right)^{2}-\left(\alpha_{-1}^{I}\right)^{2}\left(\alpha_{-1}^{L}\right)^{2}-\left(\alpha_{-1}^{I}\right)^{2}\left(\alpha_{-1}^{T}\right)^{2}\right. \\
\left.+\frac{1}{3}\left(\alpha_{-1}^{I}\right)^{4}\right]|0 ; k\rangle \\
|D\rangle=\left[\left(\alpha_{-1}^{L}\right)^{3} \alpha_{-1}^{T}-3 \alpha_{-1}^{L} \alpha_{-1}^{T}\left(\alpha_{-1}^{I}\right)^{2}\right]|0 ; k\rangle \\
|E\rangle=\left[\left(\alpha_{-1}^{L}\right)^{4}-6\left(\alpha_{-1}^{L}\right)^{2}\left(\alpha_{-1}^{I}\right)^{2}+\left(\alpha^{I}-1\right)^{4}\right]|0 ; k\rangle, \\
|F\rangle=\left[\alpha_{-1}^{T} \alpha_{-3}^{T}-\frac{3}{4}\left(\alpha_{-2}^{T}\right)^{2}-3\left(\alpha_{-1}^{T}\right)^{2}\left(\alpha_{-1}^{I}\right)^{2}\right. \\
\left.+\frac{1}{2}\left(\alpha_{-1}^{I}\right)^{4}\right]|0 ; k\rangle \\
|G\rangle=\left[\alpha_{-1}^{T} \alpha_{-3}^{L}+\alpha_{-1}^{L} \alpha_{-3}^{T}-\frac{3}{2} \alpha_{-2}^{T} \alpha_{-2}^{L}\right. \\
\left.\quad-6 \alpha_{-1}^{T} \alpha_{-1}^{L}\left(a_{-1}^{I}\right)^{2}\right]|0 ; k\rangle,
\end{gathered}
$$

$$
\begin{aligned}
&|H\rangle= {\left[\alpha_{-1}^{L} \alpha_{-3}^{L}-\frac{3}{4}\left(\alpha_{-2}^{L}\right)^{2}-3\left(\alpha_{-1}^{L}\right)^{2}\left(\alpha_{-1}^{I}\right)^{2}\right.} \\
&\left.+\frac{1}{2}\left(\alpha_{-1}^{I}\right)^{4}\right]|0 ; k\rangle, \\
&|I\rangle=\frac{1}{2}\left[\left(\alpha_{-1}^{T}\right)^{2} \alpha_{-2}^{L}-\left(\alpha_{-1}^{I}\right)^{2} \alpha_{-2}^{L}-\alpha_{-1}^{T} \alpha_{-1}^{L} \alpha_{-2}^{T}\right.\left.+\alpha_{-1}^{I} \alpha_{-1}^{L} \alpha_{-2}^{I}\right]|0 ; k\rangle, \\
&|J\rangle=\frac{1}{2}\left[\left(\alpha_{-1}^{L}\right)^{2} \alpha_{-2}^{T}-\left(\alpha_{-1}^{I}\right)^{2} \alpha_{-2}^{T}-\alpha_{-1}^{L} \alpha_{-1}^{T} \alpha_{-2}^{L}\right. \\
&+ \\
&\left.+\alpha_{-1}^{I} \alpha_{-1}^{T} \alpha_{-2}^{I}\right]|0 ; k\rangle .
\end{aligned}
$$

To skip lengthy details, let us just give the final result. Solving the constraint obtained from decoupling spurious states (and replacing $e^{P}$ by $e^{L}$ ), one can find the ratios among amplitudes at the leading order [4],

$$
\mathcal{T}(A): \mathcal{T}(C): \mathcal{T}(E): \mathcal{T}(H): \mathcal{T}(I)=96: 8: 2:-3:-4 \sqrt{6}
$$

For example,

$$
\mathcal{T}(A)=\mathcal{T}(6) E^{12}\left[32 \sin ^{4} \phi-160 \sin ^{4} \phi \frac{1}{E^{2}}+O\left(\frac{1}{E^{4}}\right)\right]
$$

where

$$
\begin{aligned}
\mathcal{T}(6)= & \frac{\Gamma\left(\frac{-s}{2}-1\right) \Gamma\left(\frac{-t}{2}-1\right)}{\Gamma\left(\frac{u}{2}+2\right)}+\frac{\Gamma\left(\frac{-t}{2}-1\right) \Gamma\left(\frac{-u}{2}-1\right)}{\Gamma\left(\frac{s}{2}+2\right)} \\
& +\frac{\Gamma\left(\frac{-u}{2}-1\right) \Gamma\left(\frac{-s}{2}-1\right)}{\Gamma\left(\frac{t}{2}+2\right)} .
\end{aligned}
$$

[Notice that $\mathcal{T}(6)$ has the same form as $\mathcal{T}(2)$ in Eq. (39), but the definitions of $s, t$, and $u$ depend on the mass levels.] Again, there is another nonphysical state such as $\left(\alpha_{-1}^{T}\right)^{2} \alpha_{-2}^{L}|0 ; k\rangle$ which is linearly related to these states at the leading order. A new result of this paper is the ratio among the next-to-leading-order amplitudes,

$$
\mathcal{T}(B): \mathcal{T}(D): \mathcal{T}(G): \mathcal{T}(J)=36: 11: 3: 2 \sqrt{6}
$$

For the four-point functions involving three tachyons, we have

$$
\begin{aligned}
\mathcal{T}(B)= & \mathcal{T}(6) E^{11}\left[16 \sqrt{6} \cos \phi \sin ^{3} \phi-\frac{64 \sqrt{6} \cos \phi \sin ^{3} \phi}{E^{2}}\right. \\
& \left.+O\left(\frac{1}{E^{4}}\right)\right]
\end{aligned}
$$

etc., and the ratios above are verified.

Unlike the leading-order amplitudes, there is no nonphysical state whose amplitude has a definite ratio with any physical amplitude.

The next-to-leading-order amplitudes are of order $1 / E$ smaller than the leading-order amplitudes. There are also amplitudes of order $1 / E^{2}$ smaller than the leading-order amplitudes, such as those for $|F\rangle,(|A\rangle-12|C\rangle), \sqrt{6}|I\rangle+$ $3|C\rangle$, and $(|C\rangle-4|E\rangle)$. They are not all proportional to 
each other, but they are not independent either:

$$
\begin{aligned}
\mathcal{T}(F)=\mathcal{T}(6) & E^{10}\left[8 \sin ^{2} \phi-\frac{24 \sin ^{2} \phi}{E^{2}}+O\left(\frac{1}{E^{4}}\right)\right] \\
\mathcal{T}(A-12 C)= & \mathcal{T}(6) E^{10}\left[-16\left(33 \cos ^{2} \phi+1\right) \sin ^{2} \phi\right. \\
+ & \frac{4\left(193+191 \cos (2 \phi) \sin ^{2} \phi\right.}{E^{2}} \\
+ & \left.O\left(\frac{1}{E^{4}}\right)\right], \\
\mathcal{T}(C-4 E)= & \mathcal{T}(6) E^{10}\left[\frac{-4\left(73 \cos ^{2} \phi-1\right) \sin ^{2} \phi}{3}\right. \\
+ & \frac{2\left(151+169 \cos ^{2}(2 \phi) \sin ^{2} \phi\right)}{3 E^{2}} \\
+ & \left.O\left(\frac{1}{E^{4}}\right)\right], \\
\mathcal{T}(\sqrt{6} I+3 C)= & \mathcal{T}(6) E^{10}\left[144 \cos ^{2} \phi \sin ^{2} \phi\right. \\
& -\frac{6\left(35+37 \cos ^{2}(2 \phi)\right) \sin ^{2} \phi}{E^{2}} \\
& \left.+O\left(\frac{1}{E^{4}}\right)\right] \cdot
\end{aligned}
$$

We can see that in the $1 / E$ expansion, there are only 2 degrees of freedom at the leading order which are proportional to $\cos ^{2} \phi \sin ^{2} \phi$ and $\sin ^{2} \phi$. This result is consistent with the del Giudice, di Vecchia, Fubini (DDF) gauge consideration (see below): there are only two independent states $A_{-1}^{T} A_{-3}^{T}|0 ; k\rangle$ and $A_{-2}^{T} A_{-2}^{T}|0 ; k\rangle$ at the 2 nd subleading order.

\section{COMMENTS ON THE LINEAR RELATIONS}

The fact that we can relate all amplitudes at the leading order to each other (for a given mass level) implies that it is possible to choose another basis of physical states such that there is a unique state in the basis at the leading order, with all other states in the basis being subleading. This basis was found in [11] and called the DDF gauge. A generic state in the basis looks like

$$
A_{-n_{1}}^{i_{1}} \cdots A_{-n_{m}}^{i_{m}}\left|p_{0}\right\rangle
$$

where the $A_{-n}^{i}$ 's denote DDF operators. To define the DDF operators, one has to choose a lightlike vector $k$. Here we choose $k$ to be proportional to $\left(e^{L}-e^{P}\right)$. For the state to describe a particle with momentum $p$, we should choose the parameter $p_{0}$ in (79) to be $p_{0}=p-\left(\sum_{i} n_{i}\right) k$, which is a linear combination of $e^{L}$ and $e^{P}$. The polarization $e^{i}$ of a DDF operator $A_{-n}^{i}$ can only be one of the spatial directions transverse to the momentum.
An important property of the state in the DDF gauge is that it is only composed of the creation operators $\alpha_{-n}^{i}$ and $\alpha_{-n}^{L-P}$ when we expand them in terms of the usual creation operators $\alpha_{-n}^{\mu}$. In the computation of correlation functions, we contract all Lorentz indices. Because the components in the polarization vector $e^{L-P} \equiv e^{L}-e^{P}$ are of order $\mathcal{O}\left(E^{-1}\right)$, while those of $e^{T}$ are of order $\mathcal{O}\left(E^{0}\right)$, each factor of $\alpha_{-n}^{L-P}$ contributes a relatively suppressed amplitude in the high-energy limit. The conclusion of this consideration is that a state created by $m_{1}$ DDF operators dominates over another state created by $m_{2}$ DDF operators if and only if $m_{1}>m_{2}$. The difference in their scaling behavior in the high-energy limit is simply $E^{m_{1}-m_{2}}$. Therefore, for our computation of four-point functions, the unique state at the leading order at mass level $n$ is

$$
\left(A_{-1}^{T}\right)^{n}\left|0, p_{0}\right\rangle \text {. }
$$

Correspondingly, there is a unique four-point function at the leading order for each mass level [up to an overall constant factor given by (13)].

The state at the next-to-leading order is also unique,

$$
\left(A_{-1}^{T}\right)^{n-2} A_{-2}^{T}\left|0, p_{0}\right\rangle \text {. }
$$

This implies that all four-point functions at the next-toleading order must also be proportional to each other. This is what we showed explicitly for $M^{2}=4$ and $M^{2}=6$. Less obvious is that all the ratios among the four-point functions can be algebraically derived by setting $e^{P} \rightarrow e^{L}$ in the spurious states.

At the 2nd subleading order (next to the next-to-leading order), there are two independent DDF states,

$$
\left(A_{-1}^{T}\right)^{n-3} A_{-3}^{T}\left|0, p_{0}\right\rangle \quad \text { and }\left(A_{-1}^{T}\right)^{n-4}\left(A_{-2}^{T}\right)^{2}\left|0, p_{0}\right\rangle \text {. }
$$

Unless the two states happen to have the same high-energy behavior, we do not expect all amplitudes at the 2nd subleading order to be proportional to each other. They should satisfy linear relations involving three states at a time, as each state at this order is a linear combination of the two states above plus some other states at even lower orders. This is indeed what we observed in Eqs. (75)-(78) for the mass level $M^{2}=6$. Similarly, there can be nontrivial linear relations among amplitudes at any order for sufficiently high mass levels. For example, at the next order (the 3rd subleading order), we have three independent amplitudes associated with the following DDF states:

$$
\begin{gathered}
\left(A_{-1}^{T}\right)^{n-4} A_{-4}^{T}\left|0, p_{0}\right\rangle, \quad\left(A_{-1}^{T}\right)^{n-5} A_{-2}^{T} A_{-3}^{T}\left|0, p_{0}\right\rangle, \\
\left(A_{-1}^{T}\right)^{n-6}\left(A_{-2}^{T}\right)^{3}\left|0, p_{0}\right\rangle .
\end{gathered}
$$

After we learned that these linear relations are in some sense "trivialized" in the DDF gauge, a legitimate question is whether all these infinitely many linear relations among amplitudes have anything to do with any symmetry at all. The answer to this question is not obvious, but let us try to give some hints. First, although the linear relations seem trivial in the DDF gauge, the existence of the DDF 
gauge is highly nontrivial (for example, it exists only for $D=26$ ). The nontrivial content of the linear relations is part of what made the DDF gauge possible, which tells us that string theory has the same number of degrees of freedom as a theory of massless higher spin gauge fields.

Furthermore, there is "empirical evidence" supporting a close connection between the linear relations and symmetry. The evidence is the $2 \mathrm{D}$ string theory, which has a $w_{\infty}$ symmetry dictating all scattering amplitudes [18]. The generators of the $w_{\infty}$ symmetry are vertex operators of the so-called discrete states. It was shown [9] that, in the high-energy limit, a class of zero-norm states approaches the discrete states (also via the replacement $e^{P} \rightarrow e^{L}$ ), and thus the conservation law for each discrete state is matched with the Ward identity of a zero-norm state. This observation suggests that the linear relations derived from the decoupling of zero-norm states could bear some resemblance with the algebraic structure of the hidden symmetry.

\section{GENERIC MASS LEVELS}

In this section we will give an explicit expression for the next-to-leading-order amplitudes for all mass levels up to an overall constant, and establish a remarkably simple connection between the mass levels and the functional dependence on the scattering angle $\phi$. Since all these amplitudes at the same mass level are proportional to each other, we only need to consider a representative physical state at the next-to-leading order for each mass level.

The representative physical state we choose to compute is of the form

$$
\left[\alpha_{-1}^{L}\left(\alpha_{-1}^{T}\right)^{n-1}+\sum_{m=1}^{n-1} a_{m} \alpha_{-1}^{L}\left(\alpha_{-1}^{T}\right)^{n-1-m}\left(\alpha_{-1}^{I}\right)^{m}\right]|0 ; k\rangle
$$

where the numerical parameters $a_{k}$ should be chosen such that this is a physical state. Since the state only involves creation operators at level $1\left(\alpha_{-1}^{\mu}\right)$, almost all Virasoro generators $L_{n}$ with $n>0$ trivially annihilate the state. The only exception is the term $\frac{1}{2} \alpha_{1} \cdot \alpha_{1}$ in $L_{2}$. Thus it is only the traceless condition that needs to be taken care of. One can easily convince oneself that there are sufficient parameters, $a_{k}$ 's, for this purpose. For our computation of four-point functions in the high-energy limit, the values of the $a_{k}$ 's are irrelevant. We focus our attention on the first term in (84).

The tree level four-point function is an integral of

$$
\mathcal{A}=\left|y_{12}\right|\left|y_{13}\right|\left|y_{23}\right|\left\langle V_{1}\left(y_{1}\right) V_{2}\left(y_{2}\right) V_{3}\left(y_{3}\right) V_{4}\left(y_{4}\right)\right\rangle_{D_{2}}
$$

over the moduli space. The factor $\left|y_{12}\right|\left|y_{13}\right|\left|y_{23}\right|$ is given by the ghost part of the vertices, and $V_{i}$ 's stand for the matter part. The vertex operators we will compute are of the form

$$
V_{i}\left(y_{i}\right)=\left[\prod_{a=1}^{n_{i}} \partial X^{\mu_{a}}\left(y_{i}\right)\right] e^{i k_{i} \cdot X\left(y_{i}\right)}, \quad i=1,2,3,4
$$

where normal ordering is to be carried out.

There is a useful formula [19] to evaluate this correlation function at tree level,

$$
\begin{aligned}
\left\langle\prod_{i=1}^{n} e^{i k_{i} \cdot X\left(y_{i}\right)} \prod_{a=1}^{p} \partial_{y} X^{\mu_{a}}\left(y_{a}^{\prime}\right)\right\rangle_{D_{2}} & \\
= & i C(2 \pi)^{26} \delta^{(26)}\left(\sum_{i} k_{i}\right) \prod_{i<j}\left|y_{i j}\right|^{k_{i} \cdot k_{j}} \\
& \times\left\langle\prod_{a}\left[v^{\mu_{a}}\left(y_{a}^{\prime}\right)+q^{\mu_{a}}\left(y_{a}^{\prime}\right)\right]\right\rangle_{D_{2}},
\end{aligned}
$$

where

$$
v^{\mu}(y)=-i \sum_{i} \frac{k_{i}^{\mu}}{y-y_{i}}
$$

and the $q$ 's are contracted using

$$
\left\langle q^{\mu}(y) q^{\nu}\left(y^{\prime}\right)\right\rangle=-\frac{\eta^{\mu \nu}}{\left(y-y^{\prime}\right)^{2}} .
$$

The calculation of the four-point functions is straightforward but tedious. Here we briefly describe the techniques we use. We take the usual gauge-fixing condition

$$
y_{1}=0, \quad y_{2}=1, \quad y_{3} \rightarrow \infty, \quad y_{4}=x,
$$

where $x$ is the only modular parameter to be integrated over. A four-point function is always a linear combination of integrals of the form

$$
\int_{-\infty}^{\infty} d x x^{A}(1-x)^{B}
$$

where $A$ and $B$ are some of the Mandelstam variables $s, t, u$ plus constants. This integral can be decomposed into integrals over three different regions $(-\infty, 0),(0,1)$, and $(1, \infty)$. We have

$$
\int_{0}^{1} d x x^{A}(1-x)^{B}=\frac{\Gamma(A+1) \Gamma(B+1)}{\Gamma(A+B+2)},
$$

and a change of variable $x=1-1 / x^{\prime}$ gives

$$
\int_{-\infty}^{0} d x x^{A}(1-x)^{B}=(-1)^{A} \frac{\Gamma(-A-B-1) \Gamma(A+1)}{\Gamma(-B)},
$$

and yet another change of variable $x=1 / x^{\prime}$ gives

$$
\int_{1}^{\infty} d x x^{A}(1-x)^{B}=(-1)^{B} \frac{\Gamma(-A-B-1) \Gamma(B+1)}{\Gamma(-A)} .
$$

Using the following identity for the Gamma function,

$$
\Gamma(A+1)=A \Gamma(A),
$$


we find that, for all three integrals, the effect of increasing the parameter $A$ to $A+1$ is equivalent to adding a multiplicative factor of

$$
\left(\frac{A+1}{A+B+2}\right)
$$

Thanks to this observation, we can write the four-point function in the form

$$
\begin{aligned}
\mathcal{A}= & i C(2 \pi)^{26} \delta^{(26)}\left(\sum_{i} k_{i}\right) \\
& \times\left[\int_{-\infty}^{\infty} x^{-t / 2-2}(1-x)^{-u / 2-2}\right] \mathcal{B}(s, t, u),
\end{aligned}
$$

where $\mathcal{B}(s, t, u)$ is a fraction of polynomials of $s, t, u$. The Mandelstam variables are linearly dependent:

$$
s+t+u=\sum_{i=1}^{4} m_{i}^{2}=2(n-4),
$$

where $m_{i}=\sqrt{2\left(n_{i}-1\right)}$ is the mass of the $i$ th particle, and $n=\sum_{i=1}^{4} n_{i}$ is the sum over mass levels.

The common factor [·] in Eq. (97) can be written down more explicitly as

$$
\begin{aligned}
{\left[\int_{-\infty}^{\infty} x^{-t / 2-2}(1-x)^{-u / 2-2}\right]=} & \frac{\Gamma\left(-\frac{t}{2}-1\right) \Gamma\left(-\frac{u}{2}-1\right)}{\Gamma\left(\frac{s}{2}-n+2\right)} \\
& +\frac{\Gamma\left(-\frac{s}{2}+n-1\right) \Gamma\left(-\frac{t}{2}-1\right)}{\Gamma\left(\frac{u}{2}+2\right)} \\
& +\frac{\Gamma\left(-\frac{s}{2}+n-1\right) \Gamma\left(-\frac{u}{2}-1\right)}{\Gamma\left(\frac{t}{2}+2\right)} .
\end{aligned}
$$

As a digression, we comment that the Sterling's formula

$$
\Gamma(n+1) \simeq \sqrt{2} \pi n^{n+1 / 2} e^{-n}
$$

is valid only for $n>0$. The Gamma function diverges at negative integers. The expression

$$
\Gamma(-x)=\frac{\pi}{-x \sin (\pi x) \Gamma(x)}
$$

allows us to write down a valid approximation for large negative values. However, since this common factor is common to all amplitudes, this complication is unnecessary for our purpose of examining the relations among scattering amplitudes. We only need the high-energy expansion of $\mathcal{B}$.

Let us first recall that when all vertices are at the leading order, e.g.,

$$
V_{i}=\left(\partial X^{T}\right)^{n_{i}} e^{i k_{i} \cdot X},
$$

the four-point function has a simple high-energy limit with

$$
\mathcal{B} \simeq(-1)^{n_{1}+n_{2}}(-E \sin \phi)^{n} .
$$

After lengthy calculation, we find that if the first $k$ vertices $(0 \leq k \leq 4)$ correspond to states at the next-toleading order, e.g. (84), while the rest of the four vertices are at the leading order, e.g. (102), the high-energy limit of $\mathcal{B}$ is

$$
\mathcal{B} \simeq(-1)^{n_{1}+n_{2}}(-E \sin \phi)^{n-k} \prod_{a=1}^{k}\left(-\frac{m_{a}}{2} \cos \phi\right) .
$$

We can summarize the expression above by the following rule:

$$
\begin{gathered}
\partial X^{T} \rightarrow-E \sin \phi, \\
\partial X^{L} \rightarrow-\frac{m}{2} \cos \phi .
\end{gathered}
$$

That is, apart from the common factor of the four-point function, for every factor of $\partial X^{T}$, regardless of which vertex it resides in, we associate a factor of $(-E \sin \phi)$. The association of $\partial X^{L}$ with the factor of $\left(-\frac{m}{2} \cos \phi\right)$ needs further explanation. We only considered the case when a vertex involves at most a single factor of $\partial X^{L}$. Vertices with an even number of $\partial X^{L}$ and an arbitrary number of $\partial X^{T}$ are at the leading order, and vertices with an odd number of $\partial X^{L}$ and an arbitrary number of $\partial X^{T}$ are at the next-to-leading order. The association is restricted to states either of the form (84) or of the form (102). Nevertheless, we can now write down the general expression of all next-to-leading-order amplitudes at any mass level (up to numerical constant factors).

Our factorization rules (105) and (106) can also be written in terms of the DDF operators as

$$
A_{-1}^{T} \rightarrow-E \sin \phi, \quad A_{-2}^{T} \rightarrow-\frac{m}{2} \cos \phi .
$$

In our computation, we note that, when there are more vertices involving $\partial X^{L}$, there is more cancellation in the $1 / E$ expansion of the product $\Pi(v+q)$ in (87) since the naive power counting would give $\partial X^{L} \sim E^{2}$ (which is wrong). As a result the computation is more complicated because we need to take into consideration higher and higher order terms in the $1 / E$ expansion. The many cancellations not only reduce the four-point function to a lower order, but also lead to a result which is consistent with the remarkably simple factorization rules (105) and (106). The high-energy limit of string theory demonstrates a much simpler structure than the theory at finite energy.

It is also interesting to note that, up to a sign, both the leading and next-to-leading amplitudes [see Eqs. (97), (99), (103), and (104)] depend only on the sum of mass levels $n$, instead of depending on all four numbers $\left(n_{1}, n_{2}, n_{3}, n_{4}\right)$ (assuming that we use $E$ and $\phi$ as the parameters). This is a feature valid only in the high-energy limit. Its physical meaning remains to be understood. 


\section{ACKNOWLEDGMENTS}

The authors thank Chuan-Tsung Chan, Chong-Sun Chu, Tohru Eguchi, Clifford Johnson, Hsien-chung Kao, JenChi Lee, Yutaka Matsuo, Nicolas Moeller, Shunsuke Teraguchi, Peter West, and Yi Yang for helpful discussions. This work is supported in part by the National Science Council, and the National Center for Theoretical Sciences (NSC 94-2119-M-002-001), Taiwan, R.O.C. and the Center for Theoretical Physics at National Taiwan University.

\section{APPENDIX}

Here we list our definition of the kinematic variables involved in a four-point function. In Fig. 1, we take the scattering plane to be the $X^{1}-X^{2}$ plane. The momenta of the particles are

$$
\begin{gathered}
k_{1}=\left(\sqrt{p^{2}+m_{1}^{2}},-p, 0\right), \\
k_{2}=\left(\sqrt{p^{2}+m_{2}^{2}}, p, 0\right), \\
k_{3}=\left(-\sqrt{q^{2}+m_{3}^{2}},-q \cos \phi,-q \sin \phi\right), \\
k_{4}=\left(-\sqrt{q^{2}+m_{4}^{2}}, q \cos \phi, q \sin \phi\right) .
\end{gathered}
$$

They satisfy $k_{i}^{2}=-m_{i}^{2}$. In the high-energy limit, the Mandelstam variables are

$$
\begin{gathered}
s \equiv-\left(k_{1}+k_{2}\right)^{2} \equiv 4 E^{2}, \\
t \equiv-\left(k_{2}+k_{3}\right)^{2}, \\
u \equiv-\left(k_{1}+k_{3}\right)^{2} .
\end{gathered}
$$

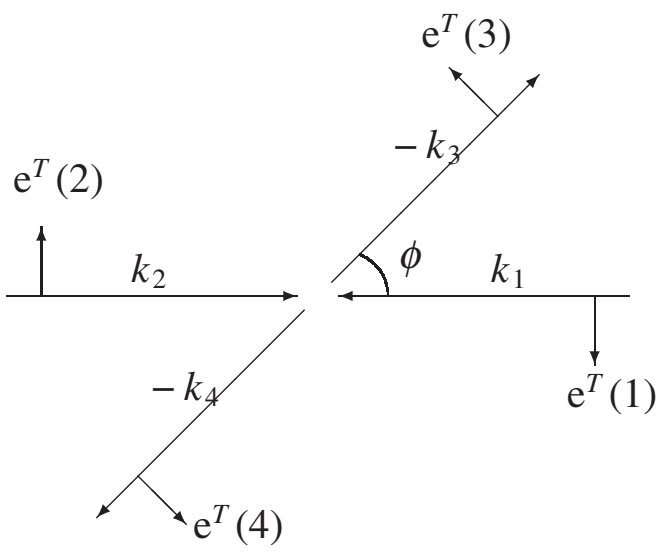

FIG. 1. Kinematic variables in the center of mass frame.

The polarization vectors for the four particles are

$$
\begin{array}{cc}
e^{L}(1)=\frac{1}{m_{1}}\left(p,-\sqrt{p^{2}+m_{1}^{2}}, 0\right), & e^{T}(1)=(0,0,-1), \\
e^{L}(2)=\frac{1}{m_{2}}\left(p, \sqrt{p^{2}+m_{2}^{2}}, 0\right), & e^{T}(2)=(0,0,1),
\end{array}
$$

$e^{L}(3)=\frac{1}{m_{3}}\left(-q,-\sqrt{q^{2}+m_{3}^{2}} \cos \phi,-\sqrt{q^{2}+m_{3}^{2}} \sin \phi\right)$,

$e^{T}(3)=(0,-\sin \phi, \cos \phi)$,

$$
e^{L}(4)=\frac{1}{m_{4}}\left(-q, \sqrt{q^{2}+m_{4}} \cos \phi, \sqrt{q^{2}+m_{4}^{2}} \sin \phi\right),
$$

$e^{T}(4)=(0, \sin \phi,-\cos \phi)$.
[1] D. J. Gross and P. F. Mende, Nucl. Phys. B303, 407 (1988); Phys. Lett. B 197, 129 (1987).

[2] D. J. Gross, Phys. Rev. Lett. 60, 1229 (1988).

[3] D. J. Gross and J. L. Manes, Nucl. Phys. B326, 73 (1989).

[4] C. T. Chan and J. C. Lee, Phys. Lett. B 611, 193 (2005).

[5] C. T. Chan and J. C. Lee, Nucl. Phys. B690, 3 (2004).

[6] C. T. Chan, P. M. Ho, and J. C. Lee, Nucl. Phys. B708, 99 (2005).

[7] J. C. Lee, Phys. Rev. Lett. 64, 1636 (1990); Z. Phys. C 54, 283 (1992); Phys. Lett. B 337, 69 (1994); 326, 79 (1994); Prog. Theor. Phys. 91, 353 (1994); H. C. Kao and J. C. Lee, Phys. Rev. D 67, 086003 (2003); J. C. Lee, Prog. Theor. Phys. 114, 259 (2005).

[8] C. T. Chan and J.C. Lee, Prog. Theor. Phys. 115, 229
(2006).

[9] C. T. Chan, P. M. Ho, J. C. Lee, S. Teraguchi, and Y. Yang, Nucl. Phys. B725, 352 (2005).

[10] C. T. Chan, P. M. Ho, J. C. Lee, S. Teraguchi, and Y. Yang, Phys. Rev. Lett. 96, 171601 (2006)..

[11] C. T. Chan, P. M. Ho, J. C. Lee, S. Teraguchi, and Y. Yang, hep-th/0509009.

[12] C. T. Chan, J. C. Lee, and Y. Yang, Nucl. Phys. B738, 93 (2006).

[13] C. T. Chan, P. M. Ho, J. C. Lee, S. Teraguchi, and Yi Yang, J. Phys.: Conf. Ser. 33, 367 (2006).

[14] G. W. Moore, hep-th/9305139; G. W. Moore, hep-th/ 9308052.

[15] P.C. West, "A Brief Review of the Group Theoretic 
Approach to String Theory" (Moriond 1988: Electroweak: 509); N. Moeller and P. West, Nucl. Phys. B729, 1 (2005).

[16] See, for instance, J. Isberg, U. Lindstrom, B. Sundborg, and G. Theodoridis, Nucl. Phys. B411, 122 (1994); B. Sundborg, Nucl. Phys. B, Proc. Suppl. 102, 113 (2001); E. Sezgin and P. Sundell, Nucl. Phys. B644, 303 (2002); B660, 403(E) (2003); C. S. Chu, P. M. Ho, and F. L. Lin, J. High Energy Phys. 09 (2002) 003.

[17] See, for instance, C. Fronsdal, Phys. Rev. D 18, 3624 (1978); J. Fang and C. Fronsdal, Phys. Rev. D 18, 3630 (1978); M. A. Vasiliev, in Proceedings of the 9th Marcel
Grossmann Meeting on Recent Developments in Theoretical and Experimental General Relativity, Gravitation and Relativistic Field Theories (MG 9), Rome, Italy, 2000; Fortschr. Phys. 52, 702 (2004).

[18] J. Avan and A. Jevicki, Phys. Lett. B 266, 35 (1991); I. R. Klebanov and A. M. Polyakov, Mod. Phys. Lett. A 6, 3273 (1991).

[19] J. Polchinski, An Introduction to the Bosonic String, String Theory Vol. 1 (Cambridge University Press, Cambridge, England, 1998). 